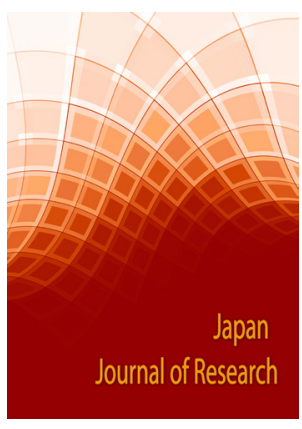

Correspondence

Bahman Zohuri

Research Associate Professor, University of New Mexico, Electrical Engineering and Computer Science Department, Albuquerque, New Mexico USA

- Received Date: 08 Aug 2019;

- Accepted Date: 19 Sep 2019;

- Publication Date: 23 Sep 2019.

Copyright

(c) 2019 Science Excel. This is an openaccess article distributed under the terms of the Creative Commons Attribution 4.0 International license.

\title{
Artificial intelligence driven resiliency with machine learning and deep learning components
}

\author{
Bahman Zohuri ${ }^{*}$ and Farhang Mossavar Rahmani ${ }^{2}$ \\ ${ }^{1}$ Research Associate Professor, University of New Mexico, Electrical Engineering and Computer Science Department, Albuquerque, \\ New Mexico, USA \\ ${ }^{2}$ Professor of Finance and Director of MBA School of Business and Management, San Diego, California, USA
}

\begin{abstract}
The future of any business from banking, e-commerce, real estate, homeland security, healthcare, marketing, the stock market, manufacturing, education, retail to government organizations depends on the data and analytics capabilities that are built and scaled. The speed of change in technology in recent years has been a real challenge for all businesses. To manage that, a significant number of organizations are exploring the Big Data $(\mathrm{BD})$ infrastructure that helps them to take advantage of new opportunities while saving costs. Timely transformation of information is also critical for the survivability of an organization. Having the right information at the right time will enhance not only the knowledge of stakeholders within an organization but also providing them with a tool to make the right decision at the right moment. It is no longer enough to rely on a sampling of information about the organizations' customers. The decision-makers need to get vital insights into the customers' actual behavior, which requires enormous volumes of data to be processed. We believe that Big Data infrastructure is the key to successful Artificial Intelligence (Al) deployments and accurate, unbiased real-time insights. Big data solutions have a direct impact and changing the way the organization needs to work with help from $\mathrm{Al}$ and its components $\mathrm{ML}$ and $\mathrm{DL}$. In this article, we discuss these topics.
\end{abstract}

\section{Introduction}

In today's growth of modern technology and the world of Robotics, significant momentum is driving the next generation of these robots that we now know as Artificial Intelligence (AI). This new generation is attracting tremendous attention of scientists and engineers. They are eager to move them to the next generation that is smarter and more cognitive, which we now call them Super Artificial Intelligence (SAI).

Every day businesses are facing the vast volume of data. Unlike before, processing this amount of data is beyond Master Data Management (MDM) to a level that we know it as Big Data (BD) that are getting around at the speed of the Internet. Since our daily operations within any organization or enterprises are expanding the Internet of Things (IoT) dealing with these data either structured or unstructured also is growing at the same speed, thus processing these data for extracting the right information for the proper knowledge growing accordingly.

With the demand of Power to make a decision with minimum risk based on Knowledge of Information from Data accumulated in Big Data (i.e. Figure1) [1] repository, we need real-time processing of the data coming to us from Omni-direction perspective. These data are centric around the $\mathrm{BD}$ and get deposited at the speed of Internet-driven mainly by IoT.
Thus, at this stage, we need to understand, what is the Big Data (BD) and why it matters when we are bringing the $\mathrm{AI}$ into play toward real-time processing of data with infrastructure around the Big data is a term that describes the large volume of data - both structured and unstructured - that inundates a business on a day-to-day basis. Big data usually has three characteristics. They are volume (the amount of data), velocity (the rate at which the data is received), and variety (types of data).

Each of these three characteristics is briefly defined here as:

1. Volume: Organizations collect data from a variety of sources, including business transactions, social media, and information from sensor or machine-to-machine data. In the past, storing it would've been a problem but new technologies (such as Hadoop) have eased the burden.

2. Velocity: Data streams in at an unprecedented speed and must be dealt with in a timely manner. RFID tags, sensors, and smart metering are driving the need to deal with torrents of data in near-real-time.

3. Variety: Data comes in all types of formats from structured, numeric data in traditional databases to unstructured text documents, email, video, audio, stock ticker data, and financial transactions. 
Although at some companies such as SAS, they consider two additional dimensions when it comes to big data:

1. Variability: In addition to the increasing velocities and varieties of data, data flows can be highly inconsistent with periodic peaks. Is something trending in social media? Daily, seasonal and eventtriggered peak data loads can be challenging to manage. Even more so with unstructured data.

2. Complexity: Today's data comes from multiple sources, which makes it difficult to link, match, cleanse and transform data across systems. However, it's necessary to connect and correlate relationships, hierarchies, and multiple data linkages or your data can quickly spiral out of control.

In a nutshell, Big Data is one of the essential concepts of our time. It is a phenomenon that has taken the world by storm. Data is growing at a compound annual growth rate of almost $60 \%$ per year.

As per studies, $70 \%$ of that vast data is unstructured. Examples of unstructured data are video files, data related to social media, etc. A look at the diversity of data itself tells the story and the challenges associated with it. Big Data is a burning topic in IT and the business world. Different people have different views and opinions about it. However, everyone agrees that [2]:

- Big Data refers to a huge amount of unstructured or semistructured data.

- Storing this mammoth detail is beyond the capacity of typical traditional database systems (relational databases).

- Legacy software tools are unable to capture, manage, and process Big Data.

Big Data is a relative term, which depends on an organization's size. Big Data is not just referring to traditional data warehouses, but it includes operational data stores that can be used for real-time applications. Also, Big Data is about finding value from the available data, which is the key to success for the business. Companies try to understand their customers better so that they can come up with more targeted products and services for them.

To do this they are increasingly adopting analytical techniques by utilizing Artificial Intelligence (AI) driven by Machine Learning (ML) and that is derived by Deep Learning (DL) for doing analysis on larger sets of data.

AI, ML and DL are defined further down in this white paper in a short format to fit this paper and their details are beyond the scope of this white paper.

To perform operations on data effectively, efficiently, and accurately, they are expanding their traditional data sets by integrating them with social media data, text analytics, and sensor data to get a complete understanding of customer's behavior.

However, it is not the amount of data that is important. It is what organizations do with the data that matters. Big data can be analyzed for insights that lead to better decisions and strategic business moves. The fact is that in today's volatile environment, every major organization needs to have the ability to adapt to disrupt while maintaining its operation quickly. That is why collecting, housing, analyzing data, and using the result of our analysis can give us the power to make a more informed decision and implement that in a timely manner.

The above statement, holistically presented in Figure 1, where partially originated by Anthony Liew 1 from Walden University in his article entitled "Understanding Data, Information, Knowledge and Their Inter-Relationships and was completed by the first two authors of this article in their volume I of book under title of A Model to Forecast Future Paradigm, Knowledge Is Power in Four Dimensions" [2].
In contrast, handling the sheer volume of data at the level of Big Data and being able to process these data for the right information in near real-time if not real-time requires a smart tool that is known as Artificial Intelligence $(\mathrm{AI})$.

Artificial intelligence (AI) is a set of technologies and building blocks, all utilizing data to unlock intelligent value across industries and businesses. It is being scaled across sectors at an enterprise level. By gathering and learning from data, cognitive systems can spot trends and provide insights to enhance workflows, response times, and client experiences and reduce the cost of operation.

The technology used in Artificial intelligence can also improve efficiency in all aspects of life. Its applications include Global Positioning Satellite (GPS), autocorrect and text predictions, automatic face detection, Object Character Recognition (OCR), image processing, stock-market, financial organization, and management of data.

Companies are further looking for AI solutions to collect, process, analyze, and gather insights from large amounts of data, helping them offer their data as a product. AI software is programmed for long hours and completes tasks faster and more efficiently.

Big Data (BD) also affects organizations across every industry. Below are examples that demonstrate how each industry can benefit from this onslaught of information:

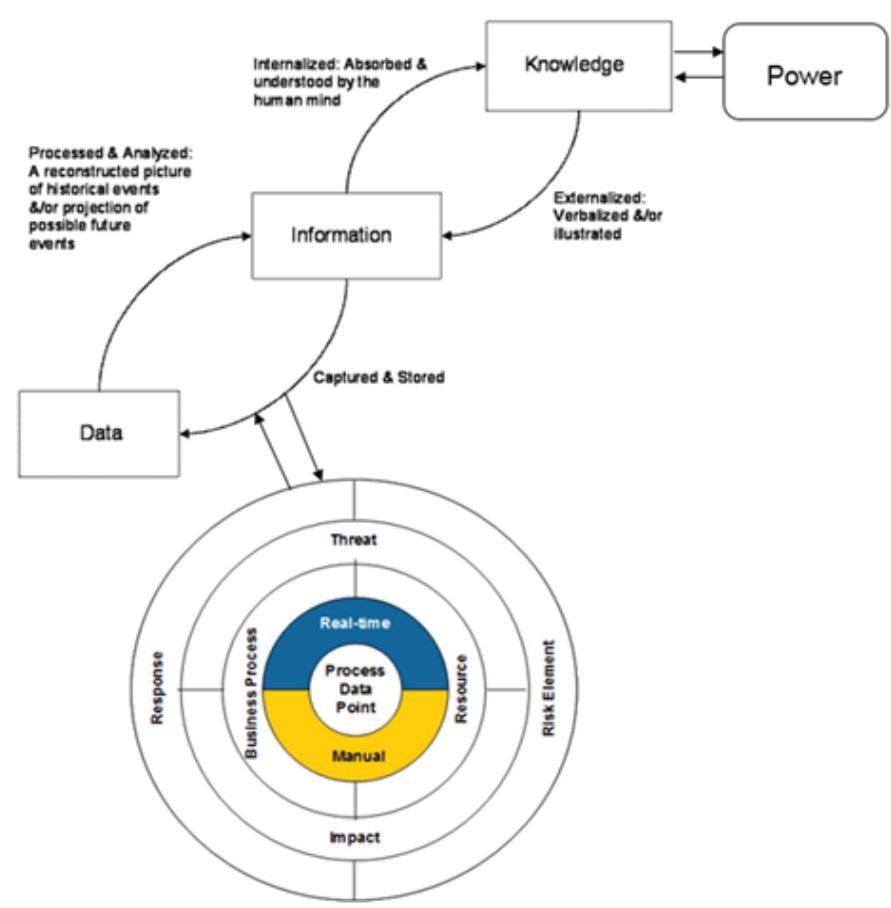

Figure 1. Depiction of Data, Information, and Knowledge is Power in Four Dimensions [1] 


\section{Government}

Big data in government -local, national and global - can have an enormous impact. With so many complex issues that we are facing today, governments are busy making sense of all the information they receive and make vital decisions that affect millions of people. Not only is it difficult to sift through all the information, but it's sometimes difficult to verify the reality of the information itself. Wrong or inaccurate information can have awful consequences.

Also, when government agencies can harness and apply analytics to their big data, they gain significant ground when it comes to managing utilities, running agencies, dealing with traffic congestion, or preventing crime. But while there are many advantages to big data, governments must also address issues of transparency and privacy.

\section{Education}

Educators armed with data-driven insight can make a significant impact on school systems, students, and curriculums. By analyzing big data, they can identify at-risk students, make sure students are making adequate progress, and can implement a better system for evaluation and support of teachers and principals.

\section{Banking}

With large amounts of information streaming in from countless sources, banks are faced with finding new and innovative ways to manage big data. While it's essential to understand customers and boost their satisfaction, it is equally important to minimize risk and fraud while maintaining regulatory compliance. Big data brings big insights, but it also requires financial institutions to stay one step ahead of the game with advanced analytics.

\section{Health Care}

Patient records. Treatment plans. Prescription information. When it comes to health care, everything needs to be done quickly, accurately - and, in some cases, with enough transparency to satisfy stringent industry regulations. When big data is managed effectively, health care providers can uncover hidden insights that improve patient care.

\section{Manufacturing}

Armed with insight that big data can provide, manufacturers can boost quality and output while minimizing waste - processes that are key in today's highly competitive market. More and more manufacturers are working in an analytics-based culture, which means they can solve problems faster and make more agile business decisions.

\section{Retail}

Customer relationship building is critical to the retail industry - and the best way to manage that is to manage big data. Retailers need to know the best way to market to customers, the most effective way to handle transactions, and the most strategic way to bring back lapsed business. Big data remains at the heart of all those things.

\section{Stock Market}

There are many techniques that are used for predicting the movement in the stock market. In recent years deep learning has been used effectively. There are different ways of using deep learning or even the latest advancements in deep learning for predicting the movement in the stock market. One example is using a Generative Adversarial Network (GAN) with Long-Short Term Memory (LSTM), a type of Recurrent Neural Network, as a generator, and a Convolutional Neural Network, CNN, as a discriminator. Another example is the use of a simple LSTM neural network with character level embeddings for stock market forecasting using only financial news as predictors [3].
However, the problem in forecasting the stock market like any other type of forecasting is that the unexpected event could change the psychology of the market and affect the outcomes. That is why no matter which techniques one uses to forecast the market; the results may not always be accurate. Boris B [4] define this process more accurately when he stated; "Accurately predicting the stock markets is a complex task as there are millions of events and pre-conditions for a particular stock to move in a particular direction. So, we need to be able to capture as many of these pre-conditions as possible. We also need to make several important assumptions: 1) markets are not 100\% random, 2) history repeats, 3) markets follow people's rational behavior, and 4) the markets are 'perfect." Therefore, in order to forecast the stock market, one has to study thousands of factors that affect the market. This is where the AI becomes valuable.

External factors may affect the stock market performance as well. Consumer confidence, geopolitical events, and the action of the Federal Reserve is just a few examples. Fed's decision especially is critical during the economic slowdown. If the economy is not doing well, the Fed can affect the outcomes by keeping the interest rates low; which might result in a rising stock market. Here again, having access to data and the ability to analyze it in a timely manner is crucial.

Of course, when it comes to the individual companies, the Selection of them is essential. Usually forecasting the performance of companies with the high market capitalization or those in high tech industries, which continuously innovate and have a reputation of high performance, is turning out to be more accurate. Here again, managing big data plays a vital role.

\section{Poverty}

Human has been trying for centuries to solve poverty across the globe without too much success. Big data and AI give governments tools to discover more effective and innovative ideas on how to address and decrease poverty across the world. It's easier to pinpoint areas with the greatest need and how those needs can be met.

It is important to remember that the primary value from big data comes not from the data in its raw form, but from the processing and analysis of it and the insights, products, and services that emerge from the analysis. The sweeping changes in big data technologies and management approaches need to be accompanied by similarly dramatic shifts in how data supports decisions and product/service innovation.

Application artificial intelligence centered around Big Data (BD) and its application in enterprises and organizations has more demand for $\mathrm{AI}$ at the level just traditional robotic action and re-action.

Associated with smart AI or in sense Super Artificial Intelligence (SAI) with capabilities of Natural Language (NL) and Neural Network (NN) [3], we need further components as sub-set of such AI that makes the processing of the data either structured or unstructured in a more accurate and faster way as they arrive with speed of electron crossnetwork into the data bank as a repository for processing purpose for the right information at the right time.

Depiction of these components concerning AI and SAI at high-level depicted in Figure-2 and they are known as:

\section{Machine Learning}

Machine Learning (ML) is a subset of AI, and it consists of the techniques that enable computers to figure things out from the data and deliver AI applications. See Figure-2

Machin learning is a branch of AI. It has been changed in the past few decades. As stated in the SAS cite [5], "Because of new computing technologies, machine learning today is not like machine learning 
of the past. It was born from pattern recognition and the theory that computers can learn without being programmed to perform specific tasks; researchers interested in artificial intelligence wanted to see if computers could learn from data.

The iterative aspect of machine learning is important because as models are exposed to new data, they are able to adapt independently. They learn from previous computations to produce reliable, repeatable decisions and results. It's a science that's not new - but one that has gained fresh momentum."

In summary, Machine Learning (ML) can also be defined as the process of solving a practical problem by:

1. Gathering dataset, and

2. Algorithmically building a statistical model based on that dataset

That statistical model is assumed to be used somehow and some way to solve the practical problem.

Machine learning as a subset of Artificial Intelligence (AI) with growth in the technology of AI has become an integral part of many commercial applications such as medical, banking, e-commerce, and research projects, but this field is not exclusive to large companies with extensive research teams. If you use software language such as Python, even as a software engineer in the field of AI any good book was written around the Python driving Machine Learning (ML) will teach you practical ways to build your own ML solutions. With growing shear of data available at the level of Big Data (BD) today.

Machine Learning applications are limited only by your imagination.

Types of Machine Learning Systems can be divided into possibly three categories, depending on whether:

1. They have been trained with humans or not,

2. If they can learn incrementally, and

3. If they work simply by comparing new data points to find data points or can detect new patterns in the data, and then will build a model, with the help of its augmentation with Deep Learning.

Learning of Machine Learning (ML) is a combination of several types, and it can be found as four major categories as 1) Supervised, 2) SemiSupervised, 3) Unsupervised, and finally 4) Reinforced type no matter, if they have been trained with humans or not.

Each of these types is briefly described as follows:

\section{Supervised}

The most successful kinds of machine learning algorithms are those that automate decision-making processes by generalizing from known examples. In this setting, which is known as supervised learning, the user provides the algorithm with pairs of inputs and desired outputs, and the algorithm finds a way to produce the desired output given an input. In particular, the algorithm is able to create an output for an input it has never seen before without any help from a human.

\section{Semi-supervised}

In Semi-Supervised learning, the dataset contains both labeled and unlabeled examples. Usually, the quantity of unlabeled examples is much higher than the number of labeled examples. The goal of a semi-supervised learning algorithm is the same as the goal of the supervised learning algorithm. The hope here is that using many unlabeled examples can help the learning algorithm to find (we might say "produce" or "compute") a better model.

\section{Unsupervised}

The third family of machine learning algorithms that we learn here is known as unsupervised learning, and we can call it as subsystems of all kinds of machine learning, where there is no known output, no teachers or trainer to instruct the learning algorithm. In unsupervised learning, the learning algorithm is just shown the input data and asked to extract knowledge from this data via its information process as depicted in Figure-2 [1].

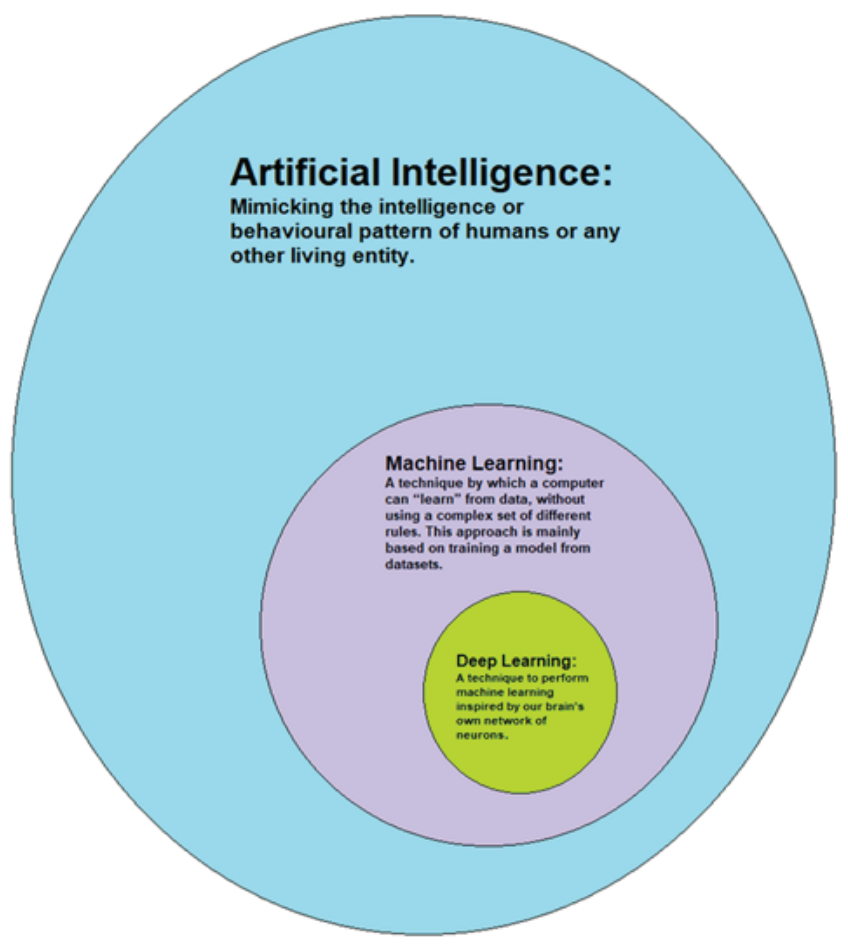

Figure 2. Presentation Artificial Intelligence in Relation with Machine Learning and Deep Learning

\section{Reinforcement type}

Reinforcement learning is a subfield of machine learning where the machine "lives" in an environment and is capable of perceiving the state of that environment as a vector of features. The machine can execute actions in every state. Different actions bring different rewards and could also move the machine to another state of the environment. The goal of a reinforcement learning algorithm is to learn a policy. A policy is a function $\mathrm{f}$ (similar to the model in supervised learning) that takes the feature vector of a state as input and outputs an optimal action to execute in that state. The action is optimal if it maximizes the expected average reward.

Reinforcement learning solves a particular kind of problems where decision making is sequential, and the goal is long-term, such as game playing, robotics, resource management, or logistics.

\section{Deep Learning}

Deep Learning (DL) is a subset of machine learning as it is illustrated in Figure-2, which enables computers to solve more complex problems. It does it through learning from experience and understand the world in terms of a hierarchy of concepts, with each concept defined through its relation to simpler concepts. Deep Learning has also been defined as learning multiple levels of representation and abstraction that help to make sense of data such as images, sound, and text. 
Put it in simple terms; deep learning is all about using neural networks with more neurons, layers, and interconnectivity. We're still a long way off from mimicking the human brain in all its complexity, but we are moving in that direction.

In summary, artificial intelligence has grabbed the center stage of business intelligence, despite having been around for decades, due to the growing pervasiveness of data, the scalability of cloud computing, the availability of AI accelerators, and the sophistication of the ML and DL algorithms. Technology of AI in its today's growth, companies like IDC technology [6], predicts that by 2019, 40\% of Digital Transformation (DX) and big data initiatives will use AI services; by $2021,75 \%$ of commercial enterprise apps will use AI, over $90 \%$ of consumers will interact with customer support bots, and over 50\% of new industrial robots will leverage AI.

Digital transformation (DX) is reaching a macroeconomic scale. Intelligent applications based on Artificial Intelligence (AI), machine learning (ML), and continual Deep Learning (DL) are the next wave of technology transforming how consumers and enterprises work, learn, and play. While data is at the core of the new digital economy, it's also about how you sense the environment and manage the data from the edge to core to cloud, analyze it in near real-time, learn from it, and then act on it to affect outcomes. The Internet of Things (IoT), mobile devices, big data, AI, ML, and DL all combine to sense and collectively learn from an environment continually. What differentiates winning organizations is how they leverage that to deliver meaningful, valueadded predictions and actions for improving industrial processes, healthcare, experiential engagement, or any other kind of enterprise decision making. AI business objectives are balanced between tactical and strategic, and they can range from improvement in operational efficiencies to increasing competitive differentiation, from maximizing existing product revenue to launching new digital revenue streams.

AI along with its sub-sets of Machine Learning (ML) and Deep Learning (DL), presents a collection of multiple technologies utilizing Tensor Processing Unit (TPU) and Graphics Processing Unit (GPU) (i.e., See Wikipedia or Internet for more information) [7] suggest by companies such as Google and Apple as a better and more enhanced processing units embedded within Supper Artificial Intelligences (SAIs) along with TensorFlow software interface hand-shaking with Python language written for command and control aspect of these SAIs are cable of enabling machines to sense, comprehend and act - and learn, either on their own and partner with its inventor human in order to augment human activities.

Super Artificial Intelligences (SAIs) driven by Artificial Neural Network (ANN) and their capability of cognitive and natural language someday may present a future matrix augmenting its human master and even surpass him/her by being Homo-Fabians and Homo-Sapiens not too far away from the present time $[3,6]$.

Artificial neural network (ANN) is a computational model based on the structure and functions of biological neural networks and is one of the main tools used in machine learning. They are brain-inspired systems that are intended to replicate the way that we humans learn. ANN belongs to a group of information-processing techniques that can be used to find knowledge, patterns, or models from a large amount of data. In general, there are three classes of Artificial Neural Networks that one needs to focus on, and they are listed below:

- $\quad$ Multilayer Perceptron (MLP).

- Convolutional Neural Network (CNN).

- Recurrent Neural Network (RNN).
These three classes of neural networks provide and augment a lot of flexibility to SAIs and have proven themselves over decades to be useful and reliable in a wide range of problems that are tasked to Super Artificial intelligences (SAIs) to handle. They also have many subtypes to help specialize them to the quirks of different framing of forecasting and prediction problems and different datasets at the level of Big Data both structured and unstructured.

With this new technology in the horizon and rapidly growing among companies and startups in Silicon Valley in particular, one is wonder if this augmentation really helps human or hurts it by taking over of so many traditional labors works away from human by industry going toward full automation in their assembly line as an example.

Compelling data reveal a discouraging truth about growth today. There has been a marked decline in the ability of traditional levers of production-capital investment and labor-to propel economic growth.

Yet, the numbers tell only part of the story. Artificial Intelligence (AI) is a new factor of production and has the potential to introduce new sources of growth, changing how work is done and reinforcing the role of people to drive growth in business [2].

\section{Artificial Intelligence Driving Economics Growth}

Artificial intelligence has significant potential to contribute to global economic activity. But widening gaps among countries, companies, and workers will need to be managed to maximize the benefits.

Accenture research on the impact of $\mathrm{AI}$ on economies reveals that AI could double annual economic growth rates in 2035 by changing the nature of work and creating a new relationship between man and machine. The impact of AI technologies on business is projected to increase labor productivity by up to 40 percent and enable people to make more efficient use of their time.

Artificial Intelligence (AI) as the new factor of production, can drive growth in at least three critical ways:

\section{Intelligent automation}

Artificial Intelligence, unlike traditional automation solutions, improve innovation, automates complex physical tasks that require adaptability and agility via cognitive and natural language as well as an artificial neural network. In other words, AI is capable of self-learning.

\section{Labor and Capital augmentation}

Existing labor and capital can be used much more effectively as Artificial Intelligence enable workers to focus on what they will do best - imagine, create and innovate and not be worried about precision and free of any flaw in the final production and assembly of products.

\section{Innovation diffusion}

One of the least-discussed benefits of Artificial Intelligence is its ability to propel innovation as it diffuses through the economy.

\section{"Artificial Intelligence heralds dramatic potential for growth for both the economy and for humans." \\ Mark Purdy, Managing Director - Economic Research, Accenture Institute for High Performance}

In conclusion, the way we see it, Artificial Intelligence (AI) in its new enhanced form, namely Super Artificial Intelligence (SAI) acting like a capital-labor hybrid. , Artificial Intelligence offers the ability to amplify and transcend the current capacity of capital and labor to propel economic growth. 
Now we need to ask the following question:

Can AI act as a complement for human creativity or SAI with its further enhancing technologies in them will become a threat to human survival? Can labor in its present form survive? Will AI take over all human activities? What will be the impact of AI on human feeling and compassion? What is going to be a long-run impact of AI on human behavior? Are we going to witness more depressions, a growing number of suicides and other side issues of AI taking over most of our daily activities?[7]

We also need to remember that Artificial Intelligence technology is evolving faster than expected. Now given the trend in today's modern technology which is already surpassing human decision making in certain Use Cases (UCs) and instances, what will the future looks like? 8

Overall, the role of artificial intelligence (AI) tools and techniques in business and the global economy is a hot topic. This is not surprising given that AI might usher in radical-arguably unprecedentedchanges in the way people live and work. The AI revolution is not in its infancy, but most of its economic impact is yet to come. The companies and institutes such as The McKinsey Global Institute looked at five broad categories of AI:

- Computer vision,

- Natural language,

- Virtual assistants,

- Robotic process automation, and

- $\quad$ Advanced machine learning.

Companies will likely use these tools to varying degrees. Some will take an opportunistic approach, testing only one technology and piloting it in a specific function (an approach our modeling calls adoption). Others might be bolder, adopting all five and then absorbing them across the entire organization (an approach we call full absorption). In between these two poles, there will be many companies at different stages of adoption; the model also captures this partial impact.

Several barriers might hinder rapid adoption and absorption. For instance, late adopters might find it difficult to generate impact from $\mathrm{AI}$, because front-runners have already captured AI opportunities and late adopters lag in developing capabilities and attracting talent.

Nevertheless, at the global average level of adoption and absorption implied by our simulation, AI has the potential to deliver additional global economic activity of around $\$ 13$ trillion by 2030 , or about 16 percent higher cumulative Gross Domestic Product (GDP) compared with today. This amounts to 1.2 percent additional GDP growth per year. If delivered, this impact would compare well with that of other general-purpose technologies throughout history (Figure-3).

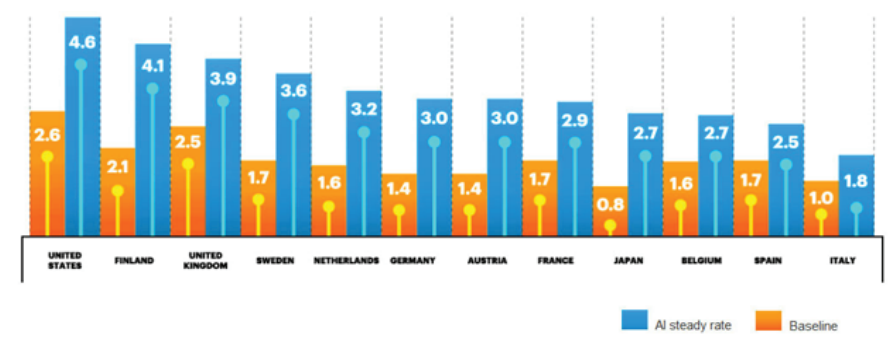

Figure 3. Annual Growth Rates in 2035 (Source: Accenture and Frontier Economics)
Note that Figure- 3 is presenting annual growth rates in 2035 of gross value added (a close approximation of GDP), comparing baseline growth in 2035 to an artificial intelligence scenario where Artificial Intelligence (AI) has been absorbed into the economy

However, take into consideration that several factors, including labor automation, innovation, and new competition, affect AI-driven productivity growth. Micro factors, such as the pace of adoption of AI, and macro factors, such as the global connectedness or labor-market structure of a country, both contribute to the size of the impact.

In summary, by acting like a capital-labor hybrid, Artificial Intelligence (AI) offers the ability to amplify and transcend the current capacity of capital and labor to propel economic growth, which is a way doubling down on growth. Our research reveals unprecedented opportunities for value creation.

Organizations and enterprises that are jumping on this train should ac upon sooner rather than later, given the momentum of technology that has been built around Artificial Intelligence and its next-generation Super Artificial Intelligence.

\section{Resiliency and Business Resilience System (BRS) with Al}

Resilience thinking is inevitably systems thinking, at least as much as sustainable development is. In fact, "when considering systems of humans and nature (social-ecological systems), it is important to consider the system as a whole." The term "resilience" originated in the 1970s in the field of ecology from the research of C.S. Holling, who defined resilience as "a measure of the persistence of systems and of their ability to absorb change and disturbance and still maintain the same relationships between populations or state variables." In short, resilience is best defined as "the ability of a system to absorb disturbances and still retain its basic function and structure." [9]

Having a right Business Resilience System (BRS) with augmentation and help from Artificial Intelligence (AI) and its sub-components such as Machine Learning (ML) and Deep Learning (DL) by building the proper intelligent models from data mining to data predictive analytics and data analytics, an organization can prevent any hostility and take over the ongoing operation that they have in place [10].

In order for Business Resilience System to function and induced advanced warning for proper action, the BRS Risk Atom and, in particular, the fourth orbit have to stay in stable status, by assessing the risk elements and responses. Thus, looking at risk assessment and understanding it is an essential fact, and it is inevitable; therefore, we need to build an intelligent model to invoke information from a variety of data available to us at more than terabyte from around the globe we are living on. These data need to be processed by Process Data Point (PDP) in the core of Risk Atom, either real time or manually. The feed point for PDP is structured on fuzzy or Boolean logic as suggested by us authors in this book. This chapter under lays foundation for Risk Atom by discussing the risk assessment and goes through process of building intelligent models, along with data mining and expert knowledge and a look at some fundamental principles that can interact with the Risk Atom. See Figure-4 [11].

One of the critical aspects of the Business Resilience System is the Risk Analysis and Risk Assessment Process. However, A critical aspect of the risk analysis process is to identify the preparedness and preventive measures in place at any point in time. Once the potential areas of high exposure to the organization are identified, additional preventative measures can be considered for implementation.

Regardless of the prevention techniques employed, possible threats that could arise inside or outside the organization need to be assessed. Although the exact nature of potential disasters or their resulting 


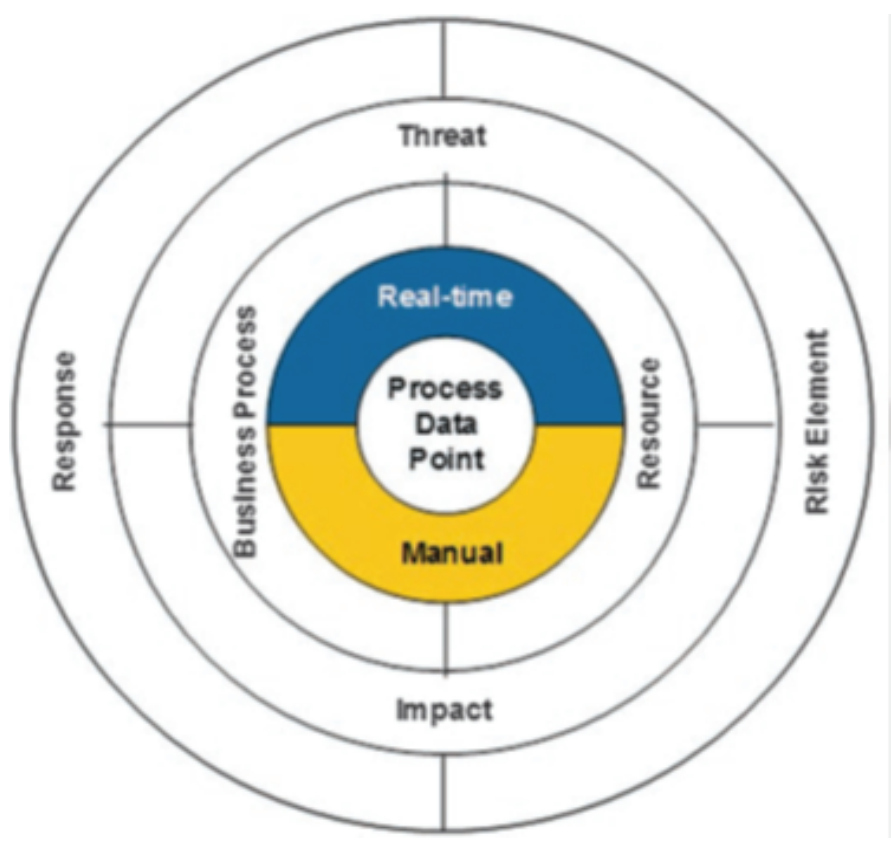

Figure 4. The Business Resilience System Risk Atom

consequences are difficult to determine, it is beneficial to perform a comprehensive risk assessment of all threats that can realistically occur to the organization. Regardless of the type of threat, the goals of business recovery planning is to ensure the safety of customers, employees, and other personnel during and following a disaster.

The relative probability of a disaster occurring should be determined. Items to consider in determining the probability of a specific disaster should include, but not be limited to:

1. Geographic location.

2. Topography of the area.

3. Proximity to major sources of power.

4. Bodies of water and airports.

5. Degree of accessibility to facilities within the organization.

6. History of local utility companies in providing uninterrupted services.

7. History of the area's susceptibility to natural threats.

8. Proximity to major highways, which transport hazardous waste and combustible products.
The above are few natural disasters that one can think of it and we are certain there is more to think about it. However, the human threat is a different ball game that our intelligent communities under the umbrella of Department of Homeland Security need to be involved and their data need to be built in a secure Master Data Management (MDM) or Big Data (BD) system with infrastructure in cloud and strong firewall, which prevents any hacker to penetrate the security of this firewall from cyber-attack perspective.

\section{Conclusion}

For any critical business process, there may be one or multiple Risk Atoms, but any Risk Atom must reflect a critical business process measure that, when "tipped," it will begin degrading process capabilities, and, if left unchecked, it will result in a disaster/destruction situation requiring the invocation of a Business Continuity Process (BCP).

A PDP can "move" through various levels of thresholds (as a result of threat manifestation) which will determine the type of business activities to be performed to remedy any foreseeable process degradation before it becomes process destruction.

\section{References}

1. B. Zohuri, and F. M. Rahmani, "A Model to Forecast Future Paradigm, Knowledge Is Power in Four Dimensions", Apple Academic Press, a CRC Press, Taylor \& Francis Group, 2019

2. Anthony Liew, Walden, Understanding Data, Information, Knowledge and Their Inter-Relationships, Journal of Knowledge Management Practice, Vol. 8, No. 2 June 2007.

3. Leonardo dos Santos Pinheiro and Mark Dras, "Stock Market Prediction with Deep Learning: A Character-based Neural Language Model for Event-based Trading." https://www.aclweb.org/anthology/U17-1001

4. https://www.sas.com/en_us/insights/analytics/machine-learning.html

5. https://towardsdatascience.com/aifortrading-2edd6fac689d\#58e2

6. B. Zohuri and M. Moghaddam, "Neural Network Driven Artificial Intelligence: Decision Making Based on Fuzzy Logic" (Computer Science, Technology and Applications: Mathematics Research Developments), Nova Publisher, 2017.

7. Ritue, Jyoti, Vice President of IDC Technology Spotlighting, "Acceleration and Operationalize AI Deployments Using Al-Optimized Infrastructure", June 2018.

8. https://en.wikipedia.org/wiki/Graphics_processing_unit

9. B. Zohuri and M. Moghaddam, Artificial Intelligence Driven by a General Neura Simulation System - Genesis, Neurology - Laboratory and Clinical Research Developments. Nova Science Pub Inc (January 29, 2018).

10. B. Zohuri and P. J. McDaniel, Electrical Brain Stimulation for the Treatment of Neurological Disorders, Apple Academic Press; 1 edition (August 27, 2019).

11. B. Zohuri and M. Moghaddam, Business Resilience System (BRS): Driven Through Boolean, Fuzzy Logics and Cloud Computation: Real and Near Real Time Analysis and Decision Making System", 1st edition, 2017, Springer Publishing Company, New York, New York. 